\title{
Absence of Ordering in a Class of Lattice Systems
}

\author{
P.-A. Vuillermot and M. V. Romerio \\ Département de Physique, Université de Neuchâtel, Switzerland
}

Received July 26, 1974; in revised form November 27, 1974

\begin{abstract}
A generalized form of the classical Bogoliubov inequality obtained by Mermin is derived for all lattice systems whose configuration manifold is a compact connected real Lie group $G$; the new inequality relates elements of $C^{\infty}(G ; \mathbb{C})$, the algebra of indefinitely differentiable complexvalued functions on $G$. We use it to prove the absence of ordering in a class of one- and two-dimensional systems defined by $G$-invariant Hamiltonians. This class contains in particular the Stanley model for ferromagnets and a lattice version of the Maier-Saupe model for nematic liquid crystals.
\end{abstract}

\section{Introduction}

It has been shown by Mermin and Wagner [1] that the isotropic Heisenberg model cannot exhibit a spontaneous magnetization at any finite temperature in one and two dimensions, provided the interactions have not too long a range. Their proof exploits an inequality due originally to Bogoliubov [2], which relates any linear self-adjoint operator, acting on some finite-dimensional Hilbert space, to two other linear operators on the same space by means of a thermal average [3].

More recently, Mermin [4] has derived an inequality of the same kind by purely classical arguments, in order to show the absence of spontaneous magnetization in various one-and two-dimensional classical spin systems; in all these cases, the inequality relates functions on a suitably chosen phase space, but is in fact only valid if certain contributions in the thermal averages vanish.

It is the object of this note to show that a generalized form of the classical Bogoliubov inequality obtained by Mermin can be derived for all lattice systems whose configuration manifold is a compact connected real Lie group $G$; the new inequality relates elements of $C^{\infty}(G ; \mathbb{C})$, the algebra of indefinitely differentiable, complex-valued functions on $G$. We use it to rule out the existence of a non-zero "order parameter" at any finite temperature in a class of one- and twodimensional systems defined by $G$-invariant Hamiltonians.

The proof of the generalized Bogoliubov inequality is given in Section 2; the main theorem concerning the absence of long-range order is proved in Section 3; Section 4 is concerned with an example which shows that the class of lattice systems considered contains most of the classical vector models generally used in the field of critical phenomena. This class includes in particular the Stanley model for ferromagnets [5] and a lattice version of the Maier-Saupe model for nematic liquid crystals $[6,7]$. 


\section{A Generalized Form for the Bogoliubov Inequality}

The $v$-dimensional lattice will be identified with $\mathbb{Z}^{v}$. We associate with every $R \in \mathbb{Z}^{v}$ a copy $G_{R}$ of a compact connected real Lie group $G$ of dimension $n$, describing the configurations of the corresponding subsystem. Let $\Lambda \subset \mathbb{Z}^{v}$ be a finite subset of lattice points and $|\Lambda|$ its cardinality; the configuration manifold associated with $\Lambda$ is then defined by the direct product

$$
G(\Lambda)=\prod_{R \in \Lambda} G_{R}
$$

of dimension $n|\Lambda|$, which is in turn compact and connected in the product topology. Let $d g$ be the bi-invariant Haar measure on $G(\Lambda)$, of total mass unity; for each function $h \in C^{\infty}(G(\Lambda) ; \mathbb{R})$, we introduce the "partition function"

$$
Z(\Lambda)=\int_{G(\Lambda)} d g \exp [-\beta h(g)]
$$

with $\beta=\left(K_{B} T\right)^{-1}>0$; then we have for all functions $\Phi \in C^{\infty}(G(\Lambda) ; \mathbb{C})$ the "thermal average"

$$
\langle\Phi\rangle_{\Lambda}=Z(\Lambda)^{-1} \int_{G(\Lambda)} d g \Phi(g) \exp [-\beta h(g)]
$$

provided $Z(\Lambda) \neq 0$.

The generalized form for the Bogoliubov inequality we will need in the next section is proved in the following

Lemma. With $N \in \mathbb{N}$, let $\left(D_{\alpha}\right)_{1 \leqq \alpha \leqq N}$ be a family of differential operators on $G(\Lambda)$, and $\left(\Phi_{\alpha}\right)_{1 \leqq \alpha \leqq N}$ a family of functions in $C^{\infty}(G(\Lambda) ; \mathbb{C})$; then for every family $\left(X_{\alpha}\right)_{1 \leqq \alpha \leqq N}$ of left-invariant complex vector fields on $G(\Lambda)$, we have

$$
\sum_{\alpha=1}^{N}\left\langle\left|D_{\alpha} \Phi_{\alpha}\right|^{2}\right\rangle_{\Lambda} \cdot \sum_{\alpha=1}^{N}\left\langle X_{\alpha}\left(\overline{X_{\alpha} h}\right)\right\rangle_{\Lambda} \geqq(\beta N)^{-1}\left|\sum_{\alpha=1}^{N}\left\langle X_{\alpha}\left(D_{\alpha} \Phi_{\alpha}\right)\right\rangle_{\Lambda}\right|^{2} .
$$

[The bar in (2.1) denotes complex conjugation.]

Proof. From the Schwarz inequality in $C^{\infty}(G(\Lambda) ; \mathbb{C})$ we get

$$
\left\langle|\Phi|^{2}\right\rangle_{A} \cdot\left\langle|\Psi|^{2}\right\rangle_{A} \geqq\left|\langle\Phi \Psi\rangle_{A}\right|^{2}
$$

for the thermal averages relating two arbitrary functions $\Phi$ and $\Psi$; in particular, we have

$$
\left\langle\left|D_{\alpha} \Phi_{\alpha}\right|^{2}\right\rangle_{\Lambda} \cdot\left\langle\left|X_{\alpha} h\right|^{2}\right\rangle_{\Lambda} \geqq\left|\left\langle\left(D_{\alpha} \Phi_{\alpha}\right)\left(X_{\alpha} h\right)\right\rangle_{\Lambda}\right|^{2}
$$

for each $\alpha$. Furthermore, since $X_{\alpha}$ is a derivation on $C^{\infty}(G(\Lambda) ; \mathbb{C})$, we can write

$$
\begin{aligned}
\exp [-\beta h]\left(D_{\alpha} \Phi_{\alpha}\right)\left(X_{\alpha} h\right)=-\beta^{-1}\left(D_{\alpha} \Phi_{\alpha}\right)\left(X_{\alpha} \exp [-\beta h]\right) \\
\quad=-\beta^{-1}\left[X_{\alpha}\left(\left(D_{\alpha} \Phi_{\alpha}\right)(\exp [-\beta h])\right)-\exp [-\beta h]\left(X_{\alpha}\left(D_{\alpha} \Phi_{\alpha}\right)\right)\right] .
\end{aligned}
$$

But for every $\Phi \in C^{\infty}(G(\Lambda) ; \mathbb{C})$ we have

$$
\int_{G(\Lambda)} d g\left(X_{\alpha} \Phi\right)(g)=0
$$


as a consequence of the left-invariance of $X_{\alpha}$; then, integrating the left- and righthand sides of (2.3) over $G(\Lambda)$, we obtain

$$
\left\langle\left(D_{\alpha} \Phi_{\alpha}\right)\left(X_{\alpha} h\right)\right\rangle_{A}=\beta^{-1}\left\langle X_{\alpha}\left(D_{\alpha} \Phi_{\alpha}\right)\right\rangle_{A} .
$$

A similar modification can be made on the second factor in (2.2), which yields

$$
\left\langle\left|X_{\alpha} h\right|^{2}\right\rangle_{A}=\beta^{-1}\left\langle X_{\alpha}\left(\overline{X_{\alpha} h}\right)\right\rangle_{A} .
$$

Substitution of (2.5) and (2.6) in (2.2) leads to

Then

$$
\left\langle\left|D_{\alpha} \Phi_{\alpha}\right|^{2}\right\rangle_{A} \cdot\left\langle X_{\alpha}\left(\overline{X_{\alpha} h}\right)\right\rangle_{A} \geqq \beta^{-1}\left|\left\langle X_{\alpha}\left(D_{\alpha} \Phi_{\alpha}\right)\right\rangle_{A}\right|^{2} .
$$

$$
\begin{aligned}
& \sum_{\alpha=1}^{N}\left\langle\left|D_{\alpha} \Phi_{\alpha}\right|^{2}\right\rangle_{A} \cdot \sum_{\alpha=1}^{N}\left\langle X_{\alpha}\left(\overline{X_{\alpha} h}\right)\right\rangle_{A} \\
& \quad \geqq \sum_{\alpha=1}^{N}\left\langle\left|D_{\alpha} \Phi_{\alpha}\right|^{2}\right\rangle_{A} \cdot\left\langle X_{\alpha}\left(\overline{X_{\alpha} h}\right)\right\rangle_{A} \geqq(\beta N)^{-1}\left|\sum_{\alpha=1}^{N}\left\langle X_{\alpha}\left(D_{\alpha} \Phi_{\alpha}\right)\right\rangle_{A}\right|^{2}
\end{aligned}
$$

which is the desired result.

Remarks. The relation (2.4) is clearly not satisfied in general if we replace $X_{\alpha}$ by an arbitrary vector field; in such a case, correction terms are obtained in the basic inequality (2.1); they are due, for example, to the contribution of the first term in (2.3), when integrated over $G(\Lambda)$. Such contributions are therefore closely related to the "surface corrections" discussed by Mermin [4].

For all examples we have in mind, it will be sufficient to choose left-invariant vector fields in order to put upper bounds on the various "order parameters" we have defined.

The connection between the inequality we have just proved and the one derived by Mermin is easily established if we observe that estimates like (2.7) can also be obtained on sympletic manifolds; the usual Bogoliubov inequality then emerges in a natural way by letting $N=1$ in (2.1) and taking for the unique $X_{\alpha}$ a vector field related to the Poisson bracket structure on these manifolds.

Now, for $R \in \mathbb{Z}^{\nu}$, let $\mathfrak{G}_{R}$ be the Lie algebra of $G_{R}$; since $G_{R}$ is compact, there exists a strictly positive definite bilinear form $B$ on $\mathfrak{G}_{R} \times \mathfrak{G}_{R}$, invariant under $\operatorname{Ad}\left(G_{R}\right)$, the adjoint representation of $G_{R}$; if $\left(X_{\alpha}^{(R)}\right)_{1 \leq \alpha \leq n}$ is a basis of $\mathfrak{G}_{R}$, let $\left(Y_{\alpha}^{(R)}\right)_{1 \leqq \alpha \leqq n}$ be the dual basis with respect to $B$, i.e. $\bar{B}\left(X_{\alpha}^{(R)}, Y_{\alpha^{\prime}}^{(R)}\right)=\delta_{\alpha \alpha^{\prime}}$ for each $\alpha$ and $\alpha^{\prime}$; then we can form the element

$$
\gamma^{(R)}=\sum_{\alpha=1}^{n} X_{\alpha}^{(R)} Y_{\alpha}^{(R)}
$$

and it is easily verified that this operator lies in the centre of the universal enveloping algebra of $\mathfrak{b}_{R}$ [8]. This implies in particular the fact that all the zonal spherical functions of $G_{R}$, defined with respect to a closed subgroup $K_{R} \subset G_{R}$, are eigenfunctions of $\gamma^{(R)}$ [9]. This result will be of crucial importance in the proof of the theorem we shall give in the next section.

In what follows, we shall never distinguish between an element $\tilde{X}$ in the Lie algebra of the group and the corresponding left-invariant vector field $X$ on the 
group itself, both being related by

$$
(X \Phi)(g)=\frac{d}{d t} \Phi(g \exp [t \tilde{X}])(t=0)
$$

for each $g \in G$ and $\Phi \in C^{\infty}(G ; \mathbb{C})[9]$.

\section{A Class of Lattice Systems without Ordering in One and Two Dimensions}

We now precisely define the class of lattice systems we want to consider. A potential in the theory is defined as an application $\chi$ which assigns to every finite subset $\Lambda \subset \mathbb{Z}^{v}$ a function $\chi(\Lambda)$ in the Banach algebra $C^{0}(G(\Lambda) ; \mathbb{R})$ of real continuous functions on $G(\Lambda)$, equipped with the norm

$$
\|\Phi\|=\max _{g \in G(\Lambda)}|\Phi(g)| .
$$

(See [10] and [11] for similar considerations concerning other types of lattice systems.)

Then the set $\mathscr{B}$ of all potentials such that

$$
\|\chi\|=\sum_{\Lambda \ni 0} \frac{\|\chi(\Lambda)\|}{|\Lambda|}<+\infty
$$

and satisfying the translation invariance

$$
\chi\left(\Lambda+R_{0}\right)\left(g_{R_{1}+R_{0}} ; \ldots ; g_{R_{|\Lambda|}+R_{0}}\right)=\chi(\Lambda)\left(g_{R_{1}}^{\prime} ; \ldots ; g_{R_{|\Lambda|}}^{\prime}\right)
$$

for each $R_{0} \in \mathbb{Z}^{v}$ and each $\left(g_{R_{1}} ; \ldots ; g_{R_{|A|}}\right) \in G(\Lambda)$ (where $g_{R_{1}}^{\prime}=g_{R_{1}+R_{0}}, \ldots, g_{R_{|A|}}^{\prime}$ $=g_{R_{|\Lambda|}+R_{0}}$ ), together with $\chi(\emptyset)=0$, inherits in a natural way the structure of a real Banach space with respect to the norm (3.1).

With $\chi^{(0)}$ and $\chi^{(1)}$ in $\mathscr{B}$, we shall restrict our attention to Hamiltonians $h \in C^{\infty}(G(\Lambda) ; \mathbb{R})$ of the form

$$
\begin{aligned}
h & =h^{(0)}+\lambda h^{(1)} \\
& =\sum_{R, R^{\prime} \in \Lambda} J\left(R-R^{\prime}\right) \chi^{(0)}\left(\left\{R, R^{\prime}\right\}\right)+\lambda \sum_{R \in \Lambda} \chi^{(1)}(\{R\})
\end{aligned}
$$

for which the norms $\left\|\chi^{(0)}\left(\left\{R, R^{\prime}\right\}\right)\right\|$ are independent of $\left\{R, R^{\prime}\right\}, J(-R)=J(R)$, $J(0)=0$ and

$$
\sum_{R \in \mathbb{Z}^{\nu}} R^{2}|J(R)|<+\infty
$$

In the first term of (3.2), each $\chi^{(0)}\left(\left\{R, R^{\prime}\right\}\right)$ is then identified with a function in $C^{\infty}(G(\Lambda) ; \mathbb{R})$; furthermore, we assume that $h^{(0)}$ is $G$-invariant, i.e.

$$
h^{(0)}\left(g_{R_{1}} g ; \ldots ; g_{R_{|A|}} g\right)=h^{(0)}\left(g_{R_{1}} \ldots g_{R_{|A|}}\right)
$$

for every $\left(g_{R_{1}} ; \ldots ; g_{R_{|\Lambda|}}\right) \in G(\Lambda)$ and every $g \in G$; at an infinitesimal level, this implies the relation

$$
\sum_{R \in \Lambda} X^{(R)} h^{(0)}=0
$$

for each copy $X^{(R)}$ of any $X$ in the Lie algebra of $G$. 
The second term in (3.2) contains a real parameter $\lambda$, which is the "symmetry breaking field" of $h^{(0)}$ in the sense that the invariance property (3.4) is no longer valid for $h^{(1)}$; however we suppose that $h^{(1)}$ is invariant under a closed subgroup $K \subset G$; finally, we assume that $\chi^{(1)}(\{R\})$, also identified in a trivial way with a function in $C^{\infty}(G(\Lambda) ; \mathbb{R})$, is a zonal spherical function of $G$ with respect to $K$.

As we shall see in Section 4, this last assumption is motivated by the fact that the thermal average of such functions is closely related to order parameters of immediate physical interest.

Now, the Condition (3.3) is sufficient to prove the existence of the Van Hove limit $f(\lambda)$ for the free energy per site

$$
f_{\Lambda}(\lambda)=-(|\Lambda| \beta)^{-1} \log \int_{G(\Lambda)} d g \exp \left[-\beta\left(h^{(0)}+\lambda h^{(1)}\right)(g)\right]
$$

for each $\lambda \in \mathbb{R}$; furthermore, for

$$
\Lambda\left(R^{(0)}\right)=\left\{R \in \mathbb{Z}^{v} ; 0 \leqq R_{i}<R_{i}^{(0)} ; i \in\{1, \ldots, v\}\right\}
$$

with $0<R^{(0)} \in \mathbb{Z}^{v}$, let $\left\{f_{\Lambda\left(R^{(0)}\right)}^{\prime}\right\}$ be the sequence of the derivatives of the free energy with respect to $\lambda$; since $f$ is concave, there exists a subsequence $\left\{\Lambda\left(R^{0}\right)_{j}\right\}_{j \in \mathbb{N}}$ such that $f_{\Lambda\left(R^{(0)}\right)_{j}}^{\prime}(\lambda)$ converges for all $\lambda \in \mathbb{R}$; indeed, on one hand, $f_{\Lambda\left(R^{(0)}\right)}^{\prime}(\lambda)$ converges to $f^{\prime}(\lambda)$ in all points where the derivative $f^{\prime}$ of $f$ exists [12]; this is always the case except for a denumerable set $\left\{\lambda_{j}\right\}_{j \in \mathbb{N}}$ of field-values; on the other hand, for these exceptional values, observing that $\left|f_{\Lambda_{\left(R^{(0)}\right)}^{\prime}}^{\prime}\left(\lambda_{j}\right)\right| \leqq\left\|\chi^{(1)}\right\| \|$ by (3.6) and (3.2), we can apply the diagonal process [13] to $f_{\Lambda\left(R^{(0)}\right)}^{\prime}\left(\lambda_{j}\right)$ for obtaining the desired subsequence; let $\eta(\lambda)$ be the limit of $f_{\Lambda\left(R^{(0)}\right)_{j}}^{\prime}(\lambda)$ [with $\eta(\lambda)=f^{\prime}(\lambda)$ almost everywhere]; we then have the following

Theorem. If we have

$$
\begin{gathered}
\gamma^{(R)} \chi^{(1)}(\{R\})=\mu \chi^{(1)}(\{R\}) \\
\text { with } \mu \neq 0 \text {, then } \lim _{\lambda \rightarrow 0} \eta(\lambda)=0 \text { if } v \leqq 2 .
\end{gathered}
$$

Proof. We assume at first that the interactions are of strictly finite range and we enclose the system in the box $\Lambda\left(R^{(0)}\right)$ [where we omit the index $j$ defining the subsequence $\Lambda\left(R^{(0)}\right)_{j}$ ] for $R^{(0)}$ sufficiently large. Let

$$
\Delta=\left\{K \in \mathbb{R}^{v} ; K_{i}=\frac{2 \pi n_{i}}{R_{i}^{(0)}} ; n_{i} \in \mathbb{Z} ;-\frac{R_{i}^{(0)}}{2}<n_{i} \leqq \frac{R_{i}^{(0)}}{2}\right\}
$$

be the corresponding first Brillouin zone; the "order parameter" related to this finite system is defined by

$$
\eta_{\Lambda\left(R^{(0)}\right)}(\lambda)=\left|\Lambda\left(R^{(0)}\right)\right|^{-1}\left\langle h^{(1)}\right\rangle_{\Lambda\left(R^{(0)}\right)}=\frac{d f_{\Lambda\left(R^{(0)}\right)}(\lambda)}{d \lambda}
$$

and we write $K \cdot R=\sum_{i=1}^{v} K_{i} R_{i}$ for every $K \in \Delta$ and every $R \in \Lambda\left(R^{(0)}\right)$. In order to put an upper bound on $\eta(\lambda)$, we now apply the inequality (2.1) with the following choice: we put $N=n$ and, for a given $K \in \Delta$, we introduce

$$
\begin{aligned}
& X_{\alpha}=\sum_{R \in \Lambda\left(R^{(0)}\right)} \exp [i K \cdot R] X_{\alpha}^{(R)} \\
& D_{\alpha}=\sum_{R \in \Lambda\left(\boldsymbol{R}^{(0)}\right)} \exp [-i K \cdot R] Y_{\alpha}^{(R)}
\end{aligned}
$$




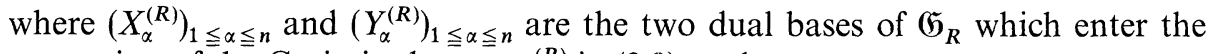
expression of the Casimir element $\gamma^{(R)}$ in (2.8), and

$$
\Phi_{\alpha}=\sum_{R \in \Lambda\left(R^{(0)}\right)} \chi^{(1)}(\{R\})
$$

for each $\alpha$; we then have successively

$\sum_{\alpha=1}^{n}\left\langle\left|D_{\alpha} \Phi_{\alpha}\right|^{2}\right\rangle_{\Lambda\left(R^{(0)}\right)}$

and

$$
=\sum_{R, R^{\prime} \in \Lambda\left(R^{(0)}\right)} \exp \left[i K \cdot\left(R-R^{\prime}\right)\right] \sum_{\alpha=1}^{n}\left\langle\left(Y_{\alpha}^{(R)} \chi^{(1)}(\{R\})\right)\left(Y_{\alpha}^{\left(R^{\prime}\right)} \chi^{(1)}\left(\left\{R^{\prime}\right\}\right)\right)\right\rangle_{\Lambda\left(R^{(0)}\right)}
$$

$$
\sum_{\alpha=1}^{n} X_{\alpha}\left(D_{\alpha} \Phi_{\alpha}\right)=\sum_{R \in \Lambda\left(R^{(0)}\right)} \gamma^{(R)} \chi^{(1)}(\{R\})=\mu \sum_{R \in \Lambda\left(R^{(0)}\right)} \chi^{(1)}(\{R\})
$$

since the functions $\chi^{(1)}(\{R\})$ are eigenfunctions of $\gamma^{(R)}$, or

$$
\left|\left\langle\sum_{\alpha=1}^{n} X_{\alpha}\left(D_{\alpha} \Phi_{\alpha}\right)\right\rangle_{\Lambda\left(R^{(0)}\right)}\right|^{2}=\mu^{2}\left|\Lambda\left(R^{(0)}\right)\right|^{2} \eta_{\Lambda\left(R^{(0)}\right)}^{2}(\lambda)
$$

by (3.7). Finally,

$$
\begin{aligned}
\sum_{\alpha=1}^{n}\left\langle X_{\alpha}\left(\overline{X_{\alpha} h}\right)\right\rangle_{\Lambda\left(R^{(0)}\right)} & \sum_{R, R^{\prime} \in \Lambda\left(R^{(0)}\right)} \exp \left[i K \cdot\left(R-R^{\prime}\right)\right] \sum_{\alpha=1}^{n}\left\langle X_{\alpha}^{(R)}\left(X_{\alpha}^{\left(R^{\prime}\right)} h^{(0)}\right)\right\rangle_{\Lambda\left(R^{(0)}\right)} \\
& +\lambda \sum_{R \in \Lambda\left(R^{(0)}\right)} \sum_{\alpha=1}^{n}\left\langle X_{\alpha}^{(R)}\left(X_{\alpha}^{(R)} \chi^{(1)}(\{R\})\right)\right\rangle_{\Lambda\left(R^{(0)}\right)} .
\end{aligned}
$$

By (2.6), this last expression is non-negative for every $K \in \Delta$; so, by adding to (3.10) the same expression where $K$ is replaced by $-K$, we have

$$
\begin{aligned}
& \sum_{\alpha=1}^{n}\left\langle X_{\alpha}\left(\overline{X_{\alpha} h}\right)\right\rangle_{\Lambda\left(R^{(0)}\right)} \leqq 2 \sum_{R, R^{\prime} \in \Lambda\left(R^{(0)}\right)} \cos K \cdot\left(R-R^{\prime}\right) \sum_{\alpha=1}^{n}\left\langle X_{\alpha}^{(R)}\left(X_{\alpha}^{\left(R^{\prime}\right)} h^{(0)}\right)\right\rangle_{\Lambda\left(R^{(0)}\right)} \\
& +2 \lambda \sum_{R \in \Lambda\left(R^{(0)}\right)} \sum_{\alpha=1}^{n}\left\langle X_{\alpha}^{(R)}\left(X_{\alpha}^{(R)} \chi^{(1)}(\{R\})\right)\right\rangle_{\Lambda\left(R^{(0)}\right)} \\
& =-2 \sum_{R, R^{\prime} \in \Lambda\left(R^{(0)}\right)}\left(1-\cos K \cdot\left(R-R^{\prime}\right)\right) \sum_{\alpha=1}^{n}\left\langle X_{\alpha}^{(R)}\left(X_{\alpha}^{\left(R^{\prime}\right)} h^{(0)}\right)\right\rangle_{\Lambda\left(R^{(0)}\right)} \\
& +2 \lambda \sum_{R \in \Lambda\left(R^{(0)}\right)} \sum_{\alpha=1}^{n}\left\langle X_{\alpha}^{(R)}\left(X_{\alpha}^{(R)} \chi^{(1)}(\{R\})\right)\right\rangle_{\Lambda\left(R^{(0)}\right)}
\end{aligned}
$$

where the last equality follows from the $G$-invariance (3.5). We then introduce the explicit form (3.2) of $h^{(0)}$ in (3.11) and insert (3.8), (3.9) and (3.11) in (2.1); since the thermal averages which appear in these expressions involve continuous functions on the compact group $G\left(\Lambda\left(R^{(0)}\right)\right)$, their absolute values are bounded from above by positive constants; the rest follows from standard manipulations [3], with the result

$$
1 \geqq \frac{(n \beta)^{-1} \mu^{2} \eta_{\Lambda\left(R^{(0)}\right)}^{2}(\lambda)}{\left|\Lambda\left(R^{(0)}\right)\right|} \sum_{K \in \Delta} \frac{1}{A K^{2}+B|\lambda|}
$$


where $A$ and $B$ are real and positive. In the thermodynamic limit $R^{(0)} \rightarrow+\infty$ (i.e. $R_{i}^{(0)} \rightarrow+\infty$ for every $i \in\{1, \ldots, v\}$ ), we obtain in particular

$$
0 \leqq \eta^{2}(\lambda) \leqq \frac{\beta n \pi A}{\mu^{2}} \cdot \frac{\left(\frac{B}{A}|\lambda|\right)^{\frac{1}{2}}}{\operatorname{arctg}\left(\frac{A \pi^{2}}{B|\lambda|}\right)^{\frac{1}{2}}}
$$

for $v=1$, and

$$
0 \leqq \eta^{2}(\lambda) \leqq \frac{2 \beta n \pi A}{\mu^{2}} \cdot \frac{1}{\log \left(\frac{A \pi^{2}}{B|\lambda|}+1\right)^{\frac{1}{2}}}
$$

if $v=2$; this implies $\lim _{\lambda \rightarrow 0} \eta(\lambda)=0$; since the two inequalities (3.12) and (3.13) remain valid for interactions satisfying (3.3), the theorem is proved.

\section{An Example}

We now present a simple example which shows that the class of lattice systems considered in the preceding section contains most of the classical vector models generally used in the field of critical phenomena; suppose we have at every $R \in \mathbb{Z}^{v}$ a copy $\mathbb{R}_{R}^{D}$ of the $D$-dimensional Euclidean space $\mathbb{R}^{D}(D \geqq 3)$ in which we consider the faithful representation of $G=S O(D)$ by orthogonal matrices. We choose in this case $K=S O(D-1)$ as closed subgroup of $G$, realized as the isotropy subgroup of a unit-vector $\boldsymbol{n}$ given in $\mathbb{R}^{D}$; the zonal spherical functions of $G$ defined with respect to $K$ are, in this case, when considered on the unit-sphere $S^{D-1}$ $\cong S O(D) / S O(D-1)$ the Gegenbauer polynomials $C_{l}^{\frac{D-2}{2}}$, with $l=0,1,2, \ldots[13]$. The chosen Hamiltonian is

$h\left(g_{R_{1}} ; \ldots ; g_{R_{|A|} \mid}\right)=\sum_{R, R^{\prime} \in \Lambda} J\left(R-R^{\prime}\right) C_{l}^{\frac{D-2}{2}}\left(g_{R}^{-1} \boldsymbol{n} \cdot g_{R^{\prime}}^{-1} \boldsymbol{n}\right)+\lambda \sum_{R \in A} C_{l}^{\frac{D-2}{2}}\left(\boldsymbol{n} \cdot g_{R} \boldsymbol{n}\right)$

with $l \neq 0$ and a "symmetry breaking field" in the direction of $\boldsymbol{n}$; this model can be interpreted as a classical spin model; we have in this case $\mu=l(l+D-2) \neq 0$ [13], and the "order parameter" which vanishes in the thermodynamic limit for $\nu \leqq 2$ and $\lambda \rightarrow 0$ is

$$
\eta_{\Lambda}(\lambda)=|\Lambda|^{-1}\left\langle\sum_{R \in A} C_{l}^{\frac{D-2}{2}}\left(\boldsymbol{n} \cdot g_{R} \boldsymbol{n}\right)\right\rangle_{\Lambda} .
$$

In particular for $l=1$, we have $C_{l}^{\frac{D-2}{2}}(X)=(D-2) X$ and we obtain the Stanley model for ferromagnets which is therefore never ferromagnetic in one and two dimensions.

For $D=3$, the Gegenbauer polynomials reduce to the Legendre polynomials $P_{l}$ and (4.1), (4.2) give respectively

$$
h\left(g_{R_{1}} ; \ldots ; g_{R_{|A|}}\right)=\sum_{R, R^{\prime} \in A} J\left(R-R^{\prime}\right) P_{l}\left(g_{R}^{-1} n \cdot g_{R^{\prime}}^{-1} \boldsymbol{n}\right)+\lambda \sum_{R \in A} P_{l}\left(\boldsymbol{n} \cdot g_{R} \boldsymbol{n}\right)
$$


and

$$
\eta_{\Lambda}(\lambda)=|\Lambda|^{-1}\left\langle\sum_{R \in \Lambda} P_{l}\left(\boldsymbol{n} \cdot g_{R} \boldsymbol{n}\right)\right\rangle_{\Lambda} .
$$

In particular if $l=1$, we obtain the classical Heisenberg model which has been discussed by Mermin in [4]; if $l=2$, we have with (4.3) a lattice version of the Maier-Saupe model for nematic liquid crystals $([6,7,15])$, in which the vectors $g_{R} n$ are interpreted as the "directors" of long thin rods whose centre-of-mass are fixed at the lattice sites; this model then cannot exhibit "nematic ordering" if $v \leqq 2$.

\section{Concluding Remark}

We have shown in a rigorous way how the symmetry properties of some classical Hamiltonian systems can be used to rule out the existence of "conventional" long-range order. The main ingredients are the relations (2.4) and (3.5); the first one allows the elimination of Mermin's surface corrections whereas the second one is necessary for the validity of (3.11). Another application of the inequality (2.1) concerning the study of long-range order in some anisotropic vector models of finite thickness will be published separately [15].

\section{References}

1. Mermin, N.D., Wagner, H.: Phys. Rev. Letters 17, 1133 (1966)

2. Bogoliubov, N. N.: Phys. Abhandl. S.U. 6, 113 (1962)

3. Ruelle, D.: Statistical Mechanics, rigorous results, p. 129-134. New York: Inc. W. A. Benjamin 1969

4. Mermin, N.: J. Math. Phys. 8, 1061 (1967)

5. Stanley,H.E.: Phys. Rev. 179, 570 (1969)

6. Maier, W., Saupe, A.: Z. Naturf. 14a, 882 (1959)

7. Romerio, M.V., Vuillermot, P.-A.: J. Phys. C: Solid St. Physics 7, 1033 (1974)

8. Bourbaki, N.: Groupes et algèbres de Lie, Chap. 1, p. 51, Proposition 11. Paris: Hermann 1960

9. Helgason, S.: Differential Geometry and Symmetric spaces, Chap. 10. New York: Academic Press 1962

10. Gallavotti, G., Miracle-Sole, S.: Commun. math. Phys. 5, 317 (1967)

11. Robinson, D. W.: Commun. math. Phys. 6, 151 (1967)

12. Fisher, M.E.: J. Math. Phys. 6, 1643, Appendix A (1965)

13. Dunford, N., Schwarz, J.T.: Linear Operators, Part I, p. 23. New York: Intersciences Publ. Inc. 1957

14. Vilenkin, N. J.: Special Functions and the Theory of Group Representations, Chap. 9. Providence: American Mathematical Society, 1968

15. De Gennes, P.G.: The Physics of Liquid Crystals. Oxford: Clarendon Press 1974

16. Vuillermot,P.-A.: Thèse, Université de Neuchâtel (1974) 